\section{Giant Carotid Pseudoaneurysm After Carotid Endarterectomy}

Robert T. Tung, M.D. ${ }^{1}$, Johannes Heyns, M.D. ${ }^{2}$

Department of Veterans Affairs, Eastern Kansas HealthCare

$$
\begin{gathered}
\text { System, Topeka, KS } \\
{ }^{1} \text { Cardiology Section } \\
{ }^{2} \text { Radiology Department }
\end{gathered}
$$

Received Dec. 8, 2019; Accepted for publication May 7, 2020; Published online July 10, 2020

\section{INTRODUCTION}

Carotid endarterectomy (CEA) is a commonly performed vascular surgery for severe, symptomatic carotid artery stenosis. Development of pseudoaneurysm (PA) following CEA is a rare complication with an incidence of less than $1 \%{ }^{1,2}$ The etiology of PA can include suture failure, degeneration of arterial wall or patch material, and infection.,3 Repair of carotid PA usually is required surgically ${ }^{4}$ or via endovascular approaches. ${ }^{5}$ Very large or giant PAs after CEA are very rare and frequently associated with symptoms of swelling, pain, rupture, infection or symptoms of transient ischemic attack (TIA) or stroke. ${ }^{6,7}$

We report a case of giant PA found incidentally many years after CEA with minimal symptoms and it was treated successfully with a stent-graft approach.

\section{CASE REPORT}

A 67-year-old African-American male with extensive medical history was admitted for alcohol detoxication. History of present illness included unintentional weight loss and excessive alcohol consumption. Previous medical history included hypertension, hyperlipidemia, emphysema, chronic hepatitis $\mathrm{C} /$ cirrhosis, and significant peripheral vascular disease (PVD). He had right CEA using the Boston Scientific ${ }^{\mathrm{TM}}$ vascular patch closure method 12 years prior for TIA and transient right eye vision loss in the setting of severe stenosis and ulceration of right internal carotid artery. Since CEA, no follow-up imaging was performed. This patient reported a "lump" in the neck to his provider, five to six years prior, describing it as without pain, discomfort, or symptoms of TIA or stroke. He also had a history of infrarenal abdominal aortic aneurysm (followed by vascular service) and claudication due to occluded right popliteal artery. He had a remote history of cigarette smoking and a family history of early coronary artery disease in his brother.

On admission, the patient was on full-dose aspirin, atorvastatin, and lisinopril. Pertinent physical examination showed a blood pressure of 163/78 $\mathrm{mmHg}$, a body mass index of $19.4 \mathrm{Kg} / \mathrm{m} 2$, and a left carotid bruit. Cardiac exam revealed a "soft" systolic murmur. His peripheral pulse in both distal lower extremities were present at "2+". Laboratory findings were significant for hyponatremia, anemia, severe thrombocytopenia (platelet count of 58,000/ml) as well as elevated liver function tests. To evaluate his left carotid bruit, a bilateral carotid artery Doppler study (CADS) showed “a very prominent plaque/scar tissue vs. thrombosed aneurysm at the right carotid artery bulb without significant carotid artery stenosis on both sides" (Figure 1). Because of multiple, active co-morbidities at that time, it was decided to purse elective computed tomography angiography (CTA) for further evaluation in outpatient settings.

\section{KANSAS JOURNAL of MEDICINE}

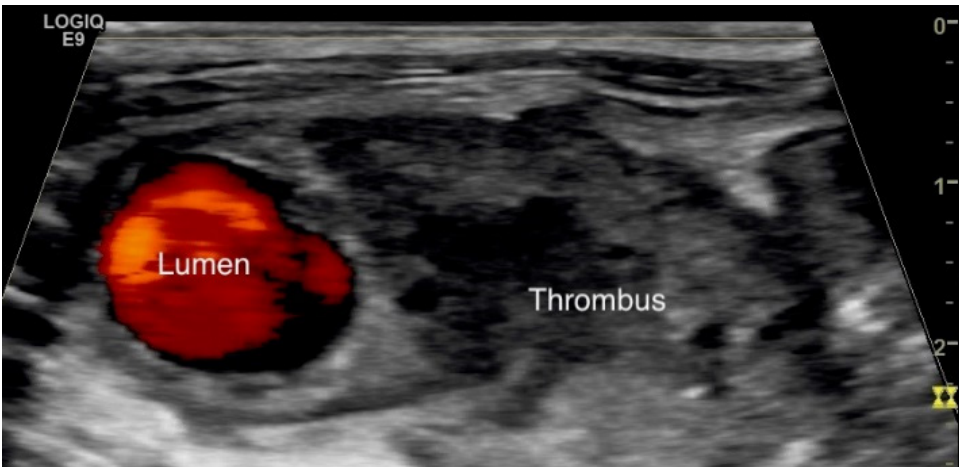

Figure l. Oblique, sagittal ultrasound view of a large pseudoaneurysm with patent carotid artery lumen and large amount of intraluminal thrombus, initially reported as "dense scar vs. thrombosed aneurysm”.

Subsequent CTA (Figures 2 - 4) confirmed a giant PA (measured at $55 \mathrm{~mm} \times 30 \mathrm{~mm})$ at distal right common carotid artery (2/3 of it was filled with intraluminal thrombus) without mass effects on native vessels or hemodynamic stenosis of right carotid artery. Thus, he was referred to vascular surgery for evaluation and management.

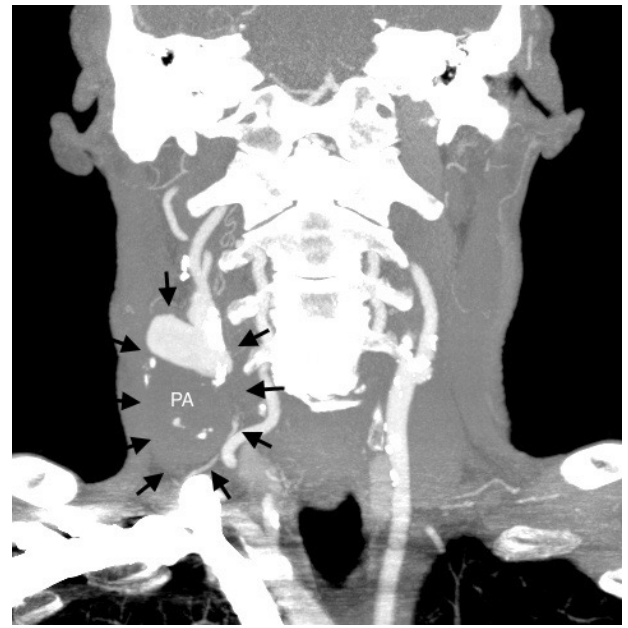

Figure 2. Coronal view of head/neck CT depicting a large pseudoaneurysm (black arrow-heads, measured at $55 \mathrm{~mm}$ in length and $30 \mathrm{~mm}$ in width) at the bifurcation of carotid artery bulb.

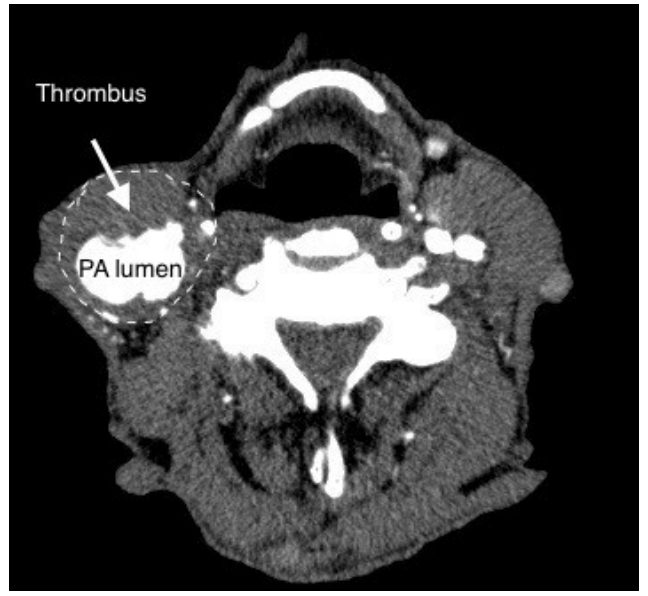

Figure 3. Sagittal CT image of pseudoaneurysm (outlined by interrupted, white line), partially filled with thrombus. 
KANSAS JOURNAL of MEDICINE GIANT CAROTID PSEUDOANEURYSM

continued.

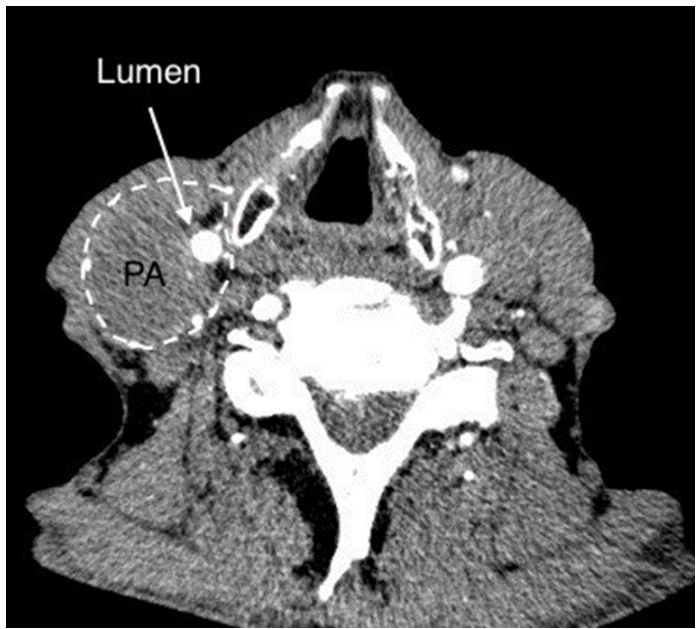

Figure 4. Sagittal CT imaging obtained inferior to the level of Figure 3. The pseudoaneurysm is outlined by interrupted, white line, and largely filled with thrombus.

The patient underwent successful endovascular closure of this very large PA using $8 \mathrm{~mm}$ x $75 \mathrm{~mm}$ Viabahn ${ }^{\oplus}$ covered-stent with cerebral protection device without any complication. He was discharged on clopidogrel for 12 months followed by baby aspirin daily along with his statins and antihypertensive medication. At his 20-month follow-up after the PA repair, there was complete resolution of the "lump" on the right side of his neck. His follow-up CADS showed the stent-graft was widely patent with resolution of his pseudoaneurysm (Figures 5 and 6).

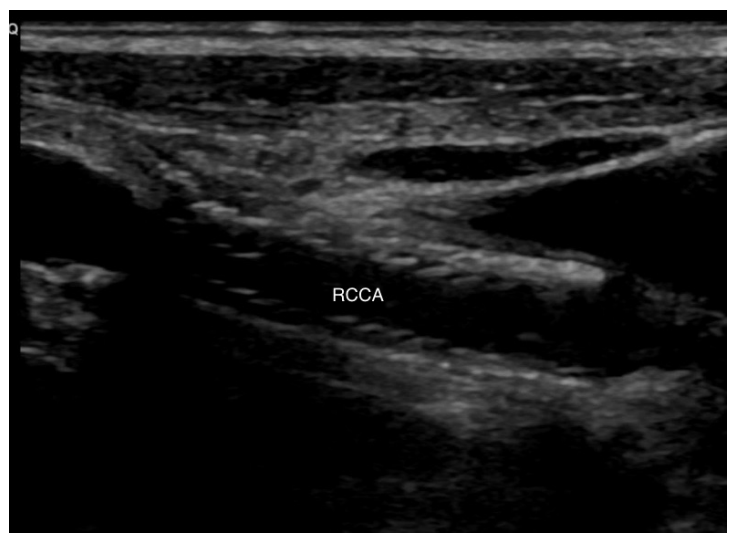

Figure 5. Sagittal view of right common carotid artery on follow-up ultrasound study. The struts of stent-graft are clearly visible.

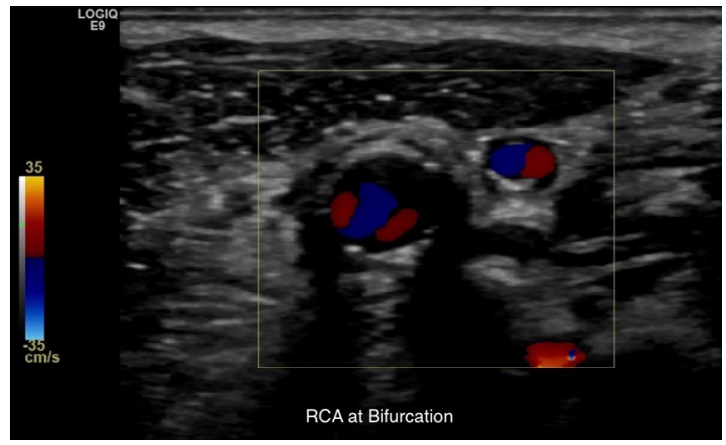

Figure 6. Transverse view of the right carotid artery at bifurcation during on follow-up ultrasound.

\section{DISCUSSION}

Carotid PA after CEA is a rare occurrence with a reported incidence of $0.3-0.4 \% .^{1,2}$ Giant PA is even rarer but a potentially severe condition which may require urgent treatment to prevent complications. The common causes of PA after CEA include suture failure, degeneration of arterial wall or patch material, and infection. ${ }^{2,3}$ The exact etiology of PA in this patient was not clear, but his poorly controlled hypertension and known severe underlying PVD might be the major contributing factors. In this case, there was lack of follow-up CADS since his CEA and his giant carotid PA were found incidentally due to a carotid bruit on the contralateral side of his prior CEA. Clinically, he was minimally symptomatic of a "lump" in the neck for years.

Patients typically have been followed by CADS regularly after CEA. The rationale is for surveillance and detection of postintervention restenosis or disease progression in the contralateral side. The current guideline recommendation states: "Noninvasive imaging of the extracranial carotid arteries is reasonable 1 month, 6 months, and annually after CEA to assess patency and exclude the development of new or contralateral lesions". ${ }^{8}$ These recommendations are intended for detection of progression of carotid artery disease, but also would detect PA after CEA despite it is a rare occurrence. Our case suggested that routine CADS follow-up and heightened clinical suspicion of PA in patients after CEA are necessary to detect of PA at an earlier stage to avoid potentially serious complications.

\section{CONCLUSIONS}

Regular noninvasive imaging study post-CEA should be performed according to current guideline for surveillance of disease progression of carotid artery disease and detection of development of PA despite its rare occurrence. Heightened clinical suspicion of PA after CEA should allow clinicians to detect and treat this potentially serious complication at an earlier stage.

\section{REFERENCES}

Branch CL Jr, Davies CH Jr. False aneurysm complicating carotid endarterectomy. Neurosurgery 1986; 19(3):421-425. PMID: 3762890.

${ }^{2}$ Abdelhamid MF, Wall ML, Vohra RK. Carotid artery pseudoaneurysm after carotid endarterectomy: Case series and a review of the literature. Vasc Endovasc Surg 2009; 43(6):571-577. PMID: 19640914.

El-Sabrout R, Reul G, Cooley DA. Infected postcarotid endarterectomy pseudoaneurysm: Retrospective review of a series. Ann Vasc Surg 2000; 14(3):239-247. PMID 10796955.

4 Ilijevski NS, Gajin P, Neskovic V, Kolar J, Radak D. Postendarterectomy common carotid artery pseudoaneurysm. Vascular 2006; 14(3):177-180. PMID: 16956493.

Briguori C, Selvetella L, Baldassarre MP. Endovascular repair of a carotid pseudoaneurysm with Fluency Plus stent graft implantation. J Invasive Cardiol 2007; 19(9):E254-E257. PMID: 17827513.

${ }^{6}$ Gupta K, Dougherty K, Hermman H, Krajcer Z. Endovascular repair of a giant carotid pseudoaneurysm with the use of Viabahn stent graft. Catheter Cadiovasc Interv 2004; 62(1):64-68. PMID: 15103606.

Defillo A, Zelensky A, Pulivarthi S, et al. Non-infected carotid artery pseudoaneurysm 29 years after endarterectomy, endovascular management with covered stent. J Neurosurg Sci 2012; 56(2):145-149. PMID: 22617177.

${ }^{8}$ Brott TG, Halperin JL, Abbara S, et al. 2011 ASA/ACCF/AHA/AANN/ AANS/ACR/ASNR/CNS/SAIP/SCAI/SIR/SNIS/SVM/SVS guideline on the management of patients with extracranial carotid and vertebral artery disease: Executive Summary. Circulation 2011; 124(4):489-532. PMID: 21282505.

Keywords: pseudoaneurysm, carotid endarterectomy, vascular surgical procedure 